\title{
A Kind of Design and Application on the Internet of Things Technology
}

\author{
Cheng-Hui YANG ${ }^{1,2, a,{ }^{*}}$ \\ ${ }^{1}$ College of Electrical Engineering, Northwest University for Nationalities, Lanzhou, Gansu, China \\ ${ }^{2}$ School of Automation \& Electrical Engineering, Lanzhou Jiaotong University, China \\ ayangchenghui36@163.com \\ ${ }^{*}$ Corresponding author
}

Keywords: The Internet of Things, RFID, Electronic Tag, Reader.

\begin{abstract}
The main topic of this paper is content on campus network technology application and design, this paper introduces the concepts, development thing networking history and the technical architecture, RFID technology is regarded as a thing of the key technology, and network application in campus thing networking plays an important. Based on RFID technology composition, working principle and classification of research, analyzes the content networking technologies in campus in a variety of applications. Student attendance has been teaching is an important link in the based on RFID checking attendance system designed to facilitate student attendance teacher to student statistics, According to the design, modular thought constructs the system overall scheme, completed the RFID transceiver module, the principle diagram of system structure and function of the main modules are analyzed in detail, and the relative accept signal strength to RSSI positioning method in inside in the classroom locate the students to prevent an students to carry more than one campus card for the other students' attendance, deceive attendance system makes the lose accuracy.
\end{abstract}

\section{Introduction}

The internet of things is an important part of new generation's information technology. As its name suggests, the Internet of Things means an internet that can contact things. It's a huge net combined with the Internet which collects objects or processes that need monitoring, connecting and interacting in real time through a variety of information sensing devices and technology like sensors, RFID, global positioning system, gas sensor and so on.. Collecting all kinds of needed information from them, such like sound, light, heat, electricity, force, chemistry, biology, position etc. The internet of things aims to achieve connection between things and things, things and human, all things and internet as well. Make identification, management and control conveniently. The core technology of the Internet of Things includes RFID, WSN network, Infrared sensor, Sensor, Global Positioning System, Internet and Mobile network, network service and Industry application software. Underlying embedded device chip development is the key among these technologies, leading the development of upper reaches of the industry as a whole.

\section{Outlines for RFID}

There are two parts included in RFID, Tag and Reader. In the specific use of RFID, we inlaid with tag on the surface or inside of the objects need identifying. When the objects with electric tag come to the area that the Reader can induce, it will read out the agreed identification information carried by the tag in the non-contact manner automatically to achieve the function of articles flag information collected or identify items automatically. There are antenna and reader contained in the reader system. There are some kinds of Readers intergrading reader and antenna elements in an equipment module.

The positioning principle of RFID, according to the signal intensity of RF card received by the Reader, distance between reader and RF card can be calculated through Indoor path loss model: 
$\operatorname{PL}(d)=\operatorname{PL}\left(d_{0}\right)-10 \ln \lg \left(d / d_{0}\right)-\mathrm{X}_{\sigma}$

In the formula:

$\mathrm{d}$ refers to the distance from the tag to the reader antenna

$\mathrm{d}_{0}$ refers to the reference distance

$\mathrm{n}$ refers to environmental parameters, the general value of classroom environment is $1 \sim 4$

$\mathrm{X} \sigma$ is Normal random variable. Standard deviation is $\sigma$.

$\mathrm{PL}(\mathrm{d})$ is the signal intensity away from $\mathrm{d}$

PL $\left(\mathrm{d}_{0}\right)$ is the signal intensity away from $\mathrm{d}_{0}$

Distance $d$ can be calculated in the above equation by PL (d) read by the Reader

\section{Applications of the Internet of Things in the Campus}

The rapid development of Internet of Things technology, as well as the popularity of computer-related hardware devices promotes the pace of the E-Campus. Major universities have completed in building digital campus of different scales and have implemented. E-Campus resources integration a certain extent currently. Bringing a lot of convenience to the daily management of school, daily life or learn of both teachers and students as well. The main applications of the Internet of Things are in the following fields:

Health management of students' bodies

Information Instruction

Consumption management in schools

Campus security management

Etc.

\section{Students Attendance Management System Design}

\section{General Design of the System}

The system aims to make students with RFID chip campus card check sensing attendance automatically in classrooms without contact punching, realizing effective management of attendance cheating by the RFID positioning technology. At the same time, the reader uploads received information to the computer terminal for processing. Then the system will record the time and seating information of students in classroom accurately etc. Teachers or teaching management staff can inquire one student's attendance at a certain period of time by logging on the web through the Browser.

\section{System Structure}

The system is made up of three parts: RFID hardware system, Internet system and Middleware system. And RFID system includes Tag, Reader, System management software and Data exchange equipment. Internet system includes Computer Communication System and Network server. Middleware system is made up of Savant server, ONS server, PML server and related data software.

\section{System Principle}

RFID technology set encoding, carrier, non-contact automatic identification and communication technology in one and identifying high-speed moving objects through RF signals. It can also identify multiple tags and obtain relevant data at the same time. The main aim of the RFID technology is to achieve automated information collection of the information system and to guarantee the information management of protected objects.

Work principle: Reader antenna will emits electromagnetic wave when the objects carrying the passive electronic tag in the range of ten meters or less close to the reader. After the signal emitted by the reader received through the electronic tags on the objects, part of this electromagnetic energy carried into for its own energy to activate the electronic label, the other part is used to transmit information in the label. Microwave signal received by the reader contains all the information carried in the tags. 


\section{System Hardware Design}

\section{Reader Design}

The Reader is made up of three parts: RF Transceiver Module, MCU Master Module and Peripheral Circuits.

\section{Campus Card Design}

The main function of Campus card is to send messages to the Reader when it comes in the effective range of the Reader. And the Reader can read information by the card. Each card stores the information of every student like student ID and so on.

\section{System Software Design}

As for the forms and content of the software, the application part of the system mainly in the way of Web applications to achieve the deployment of Query System. Web page combined with the use of JSP technology, XHTML, JavaScript, XML and Database etc.

The main aim is to obtain the attendance information of students' class late, leave early etc timely so that make inspection on situations of students' attendance. Therefore, the system is supposed to contain the following functions: checking students' status, judging students' attendance types, determining time and place of students' attendance, analyzing and classifying the information of students' attendance etc.

\section{System Algorithm Achievement}

Set up base coordinate system according to the layout of classrooms. Select four reader antennas on the basis of classroom's area and the effective radiation range of the reader. Place them in different spots in the classroom. Calculate the distance from campus card to every reader by estimating the RSSI value of each antenna relative to campus cards. Then we can derive the position of campus cards in the base coordinate system through the estimated distance.

\section{Environmental Parameters}

The overall layout of the classroom is divided into five rectangular areas (Structure of the classroom is shown in the figure, three parts are for seats). Set the reference labels in the four corners of each region.RTk-3 , RT4k-2 , RT4k-1 , RT4k (k=1,2, “’”' K, the same below), the coordinate of the reference label $\mathrm{RTi}$ is $(\mathrm{Xi}, \mathrm{Yj})\left(\mathrm{j}=1,2,{ }^{\prime} \cdot \cdots 4 \mathrm{~K}\right)$, According to the location of the four reference labels, the width $\mathrm{Wk}$ of the rectangular area $\mathrm{Zk}$ and the length $\mathrm{Lk}$ of the rectangle area $\mathrm{Zk}$ is:

$$
\mathrm{Wk}=\mathrm{X} 4 \mathrm{k}-1-\mathrm{X} 4 \mathrm{k}-3
$$

$$
\mathrm{Lk}=\mathrm{Y} 4 \mathrm{k}-2-\mathrm{Y} 4 \mathrm{k}-3
$$

Set the seats number of rows in region $\mathrm{Zk}$ is $\mathrm{nk}$, and the number of lines is $\mathrm{mk}$, the width of each seat is $w$, length (including the seat spaces) of each seat is 1 , then

$$
\mathrm{w}=\mathrm{Wk} / \mathrm{mkl}=\mathrm{Lk} / \mathrm{nk}
$$

Reader antennas locates at the four corners of the classroom, A, B,C,D are measured labels, $\Delta \sim \mathbb{D}$ are reference labels. Select typical reference labels in each seat partition and establish partition reference coordinate system and obtain the reference signals intensity of each reference label for the entire coverage antenna by practical measurement. $\Delta \sim$ Nexpress reference labels RT1 RT8. RT1 RT4 determine area $\mathrm{Zi}$. Select RT1 to be the typical reference coordinate origin $(0,0)$, Set horizontal direction determined by RT3 as X-axis and vertical direction determined by RT2 and RT3 as Y-axis, and then establish a reference coordinate system. Seat width $w$ is per unit length of $X$-axis, and 1 is the per unit length of Y-axis. Written as $(\mathrm{m}-1) \mathrm{w}<\mathrm{X}<\mathrm{mw}(\mathrm{n}-1) \mathrm{l}<\mathrm{Y}<\mathrm{nl}$ Determined seats are Dmn, $\mathrm{m}=1,2 \cdots \cdots, \mathrm{mk}, \mathrm{n}=1,2{ }^{\cdots} \cdots{ }^{\prime}, \mathrm{nk}$, 


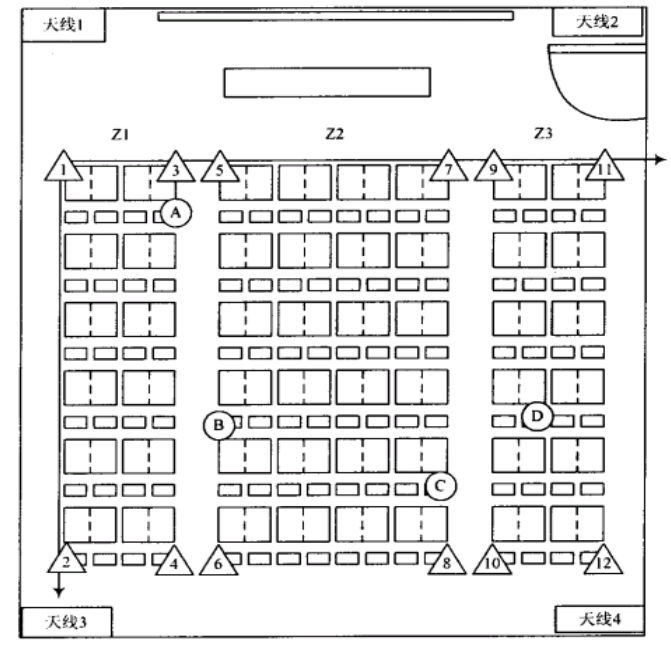

Fig.1. Classroom layout

\section{Position Determine of Campus Card}

In the basic coordinate system, set (x, y) to be the location of tested label. And set (Xi, Yi) to be the known location of the $\mathrm{i}$ reader antenna, then the distance from tested label to the $\mathrm{i}$ reader antenna is

$$
d_{i}=\sqrt{\left(X_{i}-x\right)^{2}+\left(Y_{i}-y\right)^{2}}
$$

The actual measured campus card label relative to each antenna signal intensity, through type obtained a distance value, substitution type. Two adjacent antenna equation form two yuan two equations, solving each equation in A, another node should be outside the wall; therefore, $\mathrm{A}$ is a reasonable solution.

Take all the arithmetic average value, namely the label for each of the final solution: (4) determine the tag in seating area, and converted into a corresponding seat partition reference, were calculated and integer, get campus card seat of the row and column number, and record the corresponding to the seat in the array. With all labels, we can obtain all seating placement of information.

Take the actual measured signal intensity of that campus card label relative to each antenna into formula (4) and calculate the distance value $d_{i}$,Substituting into formula(5), taking two adjacent antenna equations form binary quadratic group,solve each equation at point $\mathrm{A}$, another intersection should be outside the wall therefore,point A is reasonable solution take means of all the solutions, those are final solutions of each label:

$$
x=\sum_{i=1}^{I} x_{i} / I, y=\sum_{i=1}^{I} y_{i} / I
$$

Judge the seats area where labels locate and transform them into a corresponding seat partition reference coordinate. Calculate $\mathrm{x} / \mathrm{w}$ and $\mathrm{y} / \mathrm{l}$ apart. Rounded. Get lion and column numbers of campus card location and records to the appropriate seat array D11, D12....Dmn. Having done with all the labels, we can get all seat placement information. 


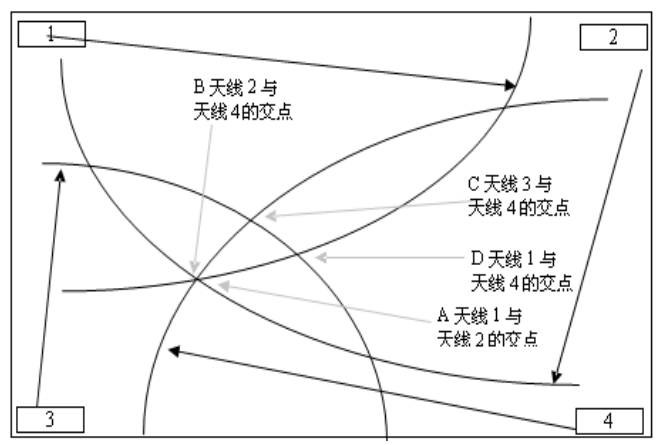

Fig.2. Coordinate label solving

\section{Workflow}

The reader gets information from RFID chip and sends it to ONS system. At the same time judging the holder's address of classroom and locate it. Then it will compare the information which have been discerned with that of stored in the Database to make sure if the holder studies in this classroom. After that, the reader will judge whether the holder be late for class or leave early. Only students who have satisfied the conditions above can check normal attendance and save the attendance information.

\section{Read Information}

During some time in class, the reader reads the information of campus cards in classroom, and judges the address that students have come in. Then produces the list of student's attendance and class address.

\section{Comparison and Check}

The reader compares the list of students' attendance with stored curriculum and class, checking if the students are having lessons in the right classrooms, and if there are students who are not supposed to study in the classroom.

\section{Check the seating information}

The reader check the seating information and records the seats with more than one cards, then save the results and output the result on the computer's screen in different colors. For example, Red means those seats with more than one card. Seats without cards will be output in black color. And green means those seats with only one card. And the absenteeism list will be listed out of the seating area on the screen.

\section{Results Deposit}

After the above steps, the data is the final results of students' attendance. Teachers and students are able to do corresponding operations according to their own permissions after logging in. For those separate students who help others hold the campus cards to cheat teachers and the system, to prevent this, instructors can compare information computer screens with these who are seating on the appropriate seats. If the data don't meet those estimated by system, That is to say there are students cheating in the attendance check, then the teacher can modify the check result manually.

\section{Conclusion}

The net of things is yet another wave of the information industry after computer, Internet and mobile communication network, and a new technology. Its strong momentum of development has been applied to various industries. However, as an emerging technology, the development of the Internet of things is still faced with series of problems. Such as Standardization system establishment, breakthrough in core technologies of independent intellectual property rights, feasibility policy implementation etc. These issues directly restrict the repaid development and popularization of the Internet of Things.

On the basis of introductions of the Internet of Things, The paper illustrates the application of radio frequency identification technology in the Internet of Things and elaborates the significance and purpose of this topic. Having studied the work principles and compositions of the radio frequency identification technology, and analyzed a variety of applications based on the Internet of Things technology in the 
campus environment, we designed the students' attendance system. The system applies intelligent means for students' attendance. Makes teaching and management more meticulous and humanized, brings great convenience to daily teaching.

There are some problems still left without good solutions because of my limited capacity during the design process. For instance, in the attendance process for students to deceive the system, I did not make a comprehensive analysis. Throughout the design process, including program identification, software and hardware design are all made by my own independently, which has trained my independent thinking as well as abilities of analyzing and solving problems, and has laid a solid foundation for future work.

\section{Acknowledgement}

This work was supported by National Natural Science Foundation of China. (Grant NO. 61263002)and by Ministry of Science and Technology of China under National 973 Basic Research Program (Grant 2011CB302805).

The project also supported by Educational reform research Funds of Northwest University for Nationalities, Northwest University for Nationalities, China. (Grant NO. JG201135). And the project also supported by two class excellent courses research Funds of Northwest University for Nationalities, Northwest University for Nationalities, China. (Grant NO. JPKC201105, Grant NO. JPKC201136).

\section{References}

[1] Wang Yali, Shi Chongyan. The era of the Internet of things on the development of School Physical Education.Journal of Nanjing Sport Institute. Natural Science Edition 2(2010).

[2] Li Wen. Technology and application in the Internet of Things, Fujian computer,9(2010).

[3] Dai Gang, Liu Yuanjie, Hao Junhua. Internet of Things RFID-based research and application. Jiangsu province post and telecommunications planning and design institute Co. Ltd.

[4] Liu Yong, Hou Rongxu. On the Internet of Things perception layer. Computer Study,5(2010).

[5] Xiong Xiangfei. Antenna research of radio frequency identification system.

[6] Hao Wenjiang, Wu Jie. Internet of Things technology applied research in public security work.

[7] Ma Jing, Wang Ping, Shi Yanxiang. The core technology RFID of the Internet of Things.

[8] Hu Rong, Lei Yuanyuan, Wang Hui. Radio frequency identification technology and its application in the Internet of Things. Jiangxi Industry Polytechnic College.

[9] Chen Guo. The basic research of network protocol based on TinyOS.

[10] Wang Wei. 125KHz RF signal recognition research.

[11] He Tongyu, Yang Shuying, Xing Ruonan. Network based on RFID technology student attendance management system design.

[12] Deng Zhaohong, Sang Qingbing. Application and thinking in the education of the Internet of Things. Journal of Wuxi Institute of Technology 9(4)8(2010). 